\title{
Outflows from Young Stars: Theory and Observation
}

\author{
Hsien Shang \\ Institute of Astronomy and Astrophysics, Academia Sinica, Taiwan
}

\begin{abstract}
Recent observations have revealed that young stellar objects are associated with jet-like structures and Herbig-Haro objects emitting at wavelengths ranging from optical lines to radio continua. These phenomena are similar in morphologies, and have mostly comparable energetics, dynamics, and kinematics. Probing such phenomena observed at various wavelengths with self-consistent physical and radiative processes arising within an inner disk-wind driven magnetocentrifugally from the circumstellar accretion disk is a challenge for confronting theory and observation of outflows. How such early outflow phase may play a role in forming planetary materials may help solve puzzles posed by meteorites. We will discuss the relevant observations, theoretical foundations for modelling approaches, magnetic structures and dynamical effects, and the connection to the early solar system.
\end{abstract}

\section{Introduction}

In the past decade, the understanding of how stars form gains tremendous boost both theoretically and observationally by the mission of the Hubble Space Telescope, and by the greatly enhanced power of the ground-based telescopes. We see stars in their infancy, and witness their formation with outflows and disks. The very frequent observational association of young stars with outflow phenomena and disks is the statement that both play important roles in the process of forming stars, and are intimately related to each other. Theoretically speaking, outflows and disks are the products of processes that transform condensed gas in clouds into stars as Suns (e.g. Shu et al 1993).

Outflow phenomena are present throughout most of the young stellar evolution, from the deeply embedded protostars, to T-Tauri stars (Class 0, I, and II). They appear in wide range of wavelengths from radio to even X-ray. They have been seen in various forms as molecular outflows in $\mathrm{CO}, \mathrm{SiO}$, and $\mathrm{H}_{2}$, atomic winds as neutral hydrogen, jets in forbidden lines from the infrared bands to the $\mathrm{UV}$ in $\mathrm{H}_{2}$, [S II], [O I], [N I], [N II], and [Fe II]. The kinematics range from low velocity molecular outflows and low velocity winds at a few tens of $\mathrm{km} / \mathrm{s}$ to high velocity jets at hundreds of $\mathrm{km} / \mathrm{s}$. Regardless of the radiative origins, either line emissions or continuum, the morphology and energetics share similar characteristics, and seem to point to one ultimate origin. To date, jets, by its highly elongated appearance or collimation, are believed to be the driving power of the outflow phenomena. 
What are jets? This question remains, and becomes more pressing. HST and Adaptic Optics have revealed images and kinematical information to a great detail: jets can be seen at optical wavelengths down to a few AUs from the sources, and knots can be resolved into bow shocks or internal working surfaces. On the theoretical fronts, models based on first-principles have reached consensus that they are magnetocentrifugally driven from near the central stars by combinations of magnetic field and rotation. Theoretically or observationally, the same question applies: what is the the emission mechanism in jets to make them as observed? Answering this question is the key to confronting theoretical modelling and comparing with observations.

In this proceedings, we focus on the theoretical picture and modeling developed based on the X-wind model (Shu et al 1994, 2000), and its processing of planetary material and connection with the early solar system.

\section{Basic Picture of The $\mathrm{X}$-winds}

The x-wind model is rooted in an evolutionary picture of how a new born star interacts with its surroundings during the important phases of accretion (Shu et al 1994, 2000). A newborn star can develop its magnetosphere as soon as it becomes sufficiently convective through the dynamo action. The magnetic fields thus generated can interact with the accretion disk where the matter comes in to feed the star. The disk is truncated by the magnetosphere and pushed away from the star, and the magnetic fields being pressed against the disk are pinched at the inner edge of the disk at the "x-point". When the field is pinched strongly enough to truncate the disk, simultaneous outflow and inflow occur along the pinched field lines from the x-point to form an "X-wind" and a "funnel flow" that carries matter to accrete onto the star. Figure 1 illustrates the schematic view in the neighborhood of the star and inner edge of the disk, and the arrows indicate the directions of the $\mathrm{x}$-winds and funnel flows. The structure of a magnetic "fan" out of the pinched magnetic fields around the x-point is shown in Fig. 9 of Shu et al (1994). In steady state, fields having foot points on both the star and the disk will synchronize the rotation of the star and the inner edge of the disk $\left(R_{\mathrm{x}}\right)$ by the balance of angular momentum.

Figure $2 a$ shows the detailed magnetic structures around the $x$-point. The magnetic fields that belong to the the $\mathrm{x}$-wind, dead zone, and the funnel flow are plotted from an actual self-consistent calculation (Ostriker, Shang, and Shu, unpublished) that imposes approximate pressure balance across the interfaces. This figure shows the magnetic field geometry where the dead zone is closed, different from the situation in Figure 1 where the dead zone field is opened. The stellar field lines shown in solid curves are opened by the $\mathrm{X}$-wind and connect at infinity to the dashed lines in the $\mathrm{X}$-wind. The dipole field lines that are rooted in the star are virtually undisturbed. Again, it is very clear that the magnetic field lines are in pressure balance around the x-region, and each component shares one-third of the trapped fluxes through the x-point. Near the boundaries where the fields mediate between the open and closed geometry, both Y-configurations are evident as the helmet streamer and reconnection ring labelled in Figure 1.

On the large scale, the hoop stresses associated with the magnetic fields slowly re-direct the matter flowing along the poloidal magnetic field ("stream- 


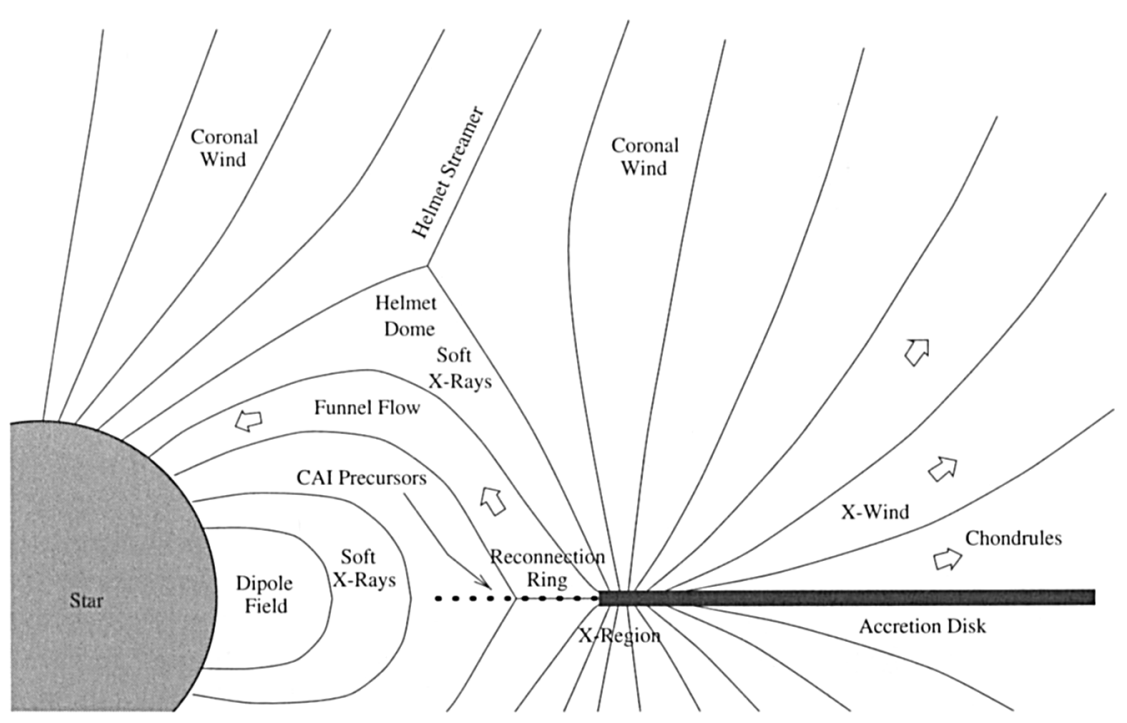

Figure 1. Schematic view of the $\mathrm{x}$-wind mode. What is shown is the innermost region of an accretion disk, truncated by stellar magnetosphere generated by early dynamo action from a newborn star.

lines") into the spin axis of the system over a long length scale by magnetic collimation. The streamlines remain very divergent, and only approach vertical at infinity. The wrapped-up field lines, on the other hand, make matter density stratified cylindrically with horizontal distances $(\varpi)$ from the rotation axes. Because $\mathrm{x}$-wind is driven from a very small region near the $\mathrm{x}$-point and completely expands to fill the space, the effect of wrapped fields and the density collimation works the strongest. The strong cylindrically stratified density profiles can be seen very clearly from a few AU from the star, and most prominent when viewing at hundreds and thousands of $\mathrm{AU}$ away from the source (see the panel for the largest scale in Figure $2 \mathrm{~b}$ ). This is the density collimation, in contrast to streamline collimation that most MHD wind models focus on.

If a unified picture can emerge, how do jets appear out of the winds driven magnetocentrifugally at more than $30^{\circ}$ from the axes? As illustrated in Figure $2 \mathrm{~b}$, the density structure of the $\mathrm{x}$-wind becomes almost cylindrically stratified from a scale as small as a few AUs out of the background very divergent streamlines. How can the density structures of the MHD winds play the trick optically through photons is the major challenge facing any theoretical MHD wind model whose matter-carrying streamlines are slowly collimated over the scale that jets have been observed. This is the basis of "optical illusion" when the optical emission lines are properly excited (Shang et al. 1998, SSG), as first proposed by Shu et al (1995). We see below that the optical and radio jets may indeed be the optical illusion of the density profiles.

These magnetic structures play very important environment effects for the $\mathrm{x}$-winds. The magnetic loops are the sites for $\mathrm{x}$-ray generation, soft and hard, in 

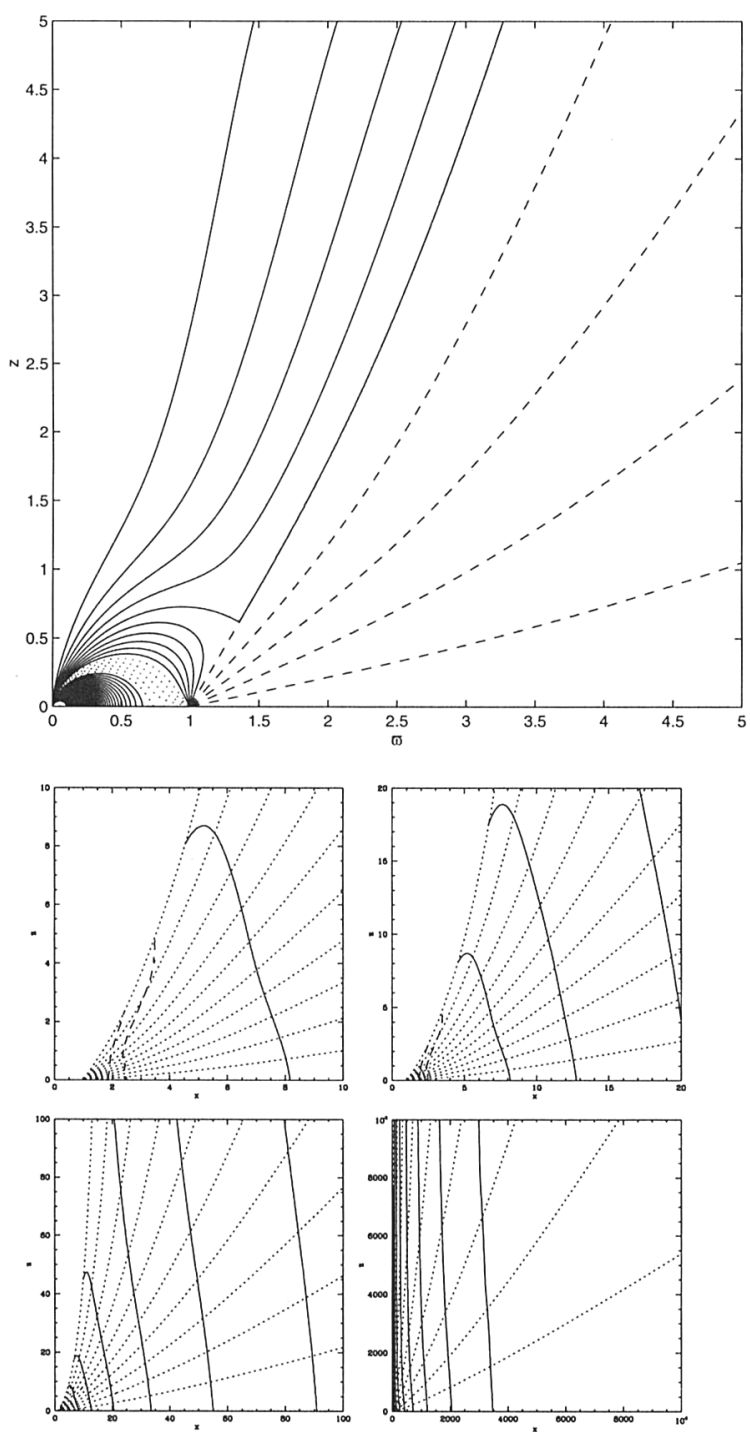

Figure 2. a. (top) The detailed magnetic structures around the xpoint. The magnetic fields that belong to the the $\mathrm{x}$-wind (dashed curves), dead zone (solid curves), and the funnel flow (dotted curves) are plotted. b. (below) Isodensity contours (solid curves) and streamlines (dotted curves) for a cold $\mathrm{x}$-wind. The contours are spaced logarithmically in intervals of $\Delta \log _{10} \rho=0.5$, and streamlines are spaced so that successive dotted lines contain an additional $10 \%$ of the total mass loss in the upper hemisphere of the flow. The loci of the Alfvén and fast surfaces are marked by dashed lines. The empty space inside the uppermost streamline, is filled with open field lines from the central star that maintains pressure balance with the $\mathrm{x}$-wind. 
quiescence and in flares. Using the solar analogy, enhanced magnetic activities can result in twisted and stretched field lines, and subject the current sheet to reconnection at the helmet streamers and the "reconnection ring". Flares go off on short time scales, strong x-ray flares arise and the events have been seen from a few protostars. UV photons, on the other hand, are generated from the hot spots where the funnel flows strike the surface of the young stars. Both radiation mechanisms are capable of ionizing and heating the wind flow with favorable penetration near the base of the wind from a slightly elevated angle above the disk. All such radiative and mechanical processes originated in the magnetic activities contribute to the optical illusion of jets.

\section{The SGSL Approach of Heating and Ionization of X-winds}

Shang et al (2002, SGSL) developed an integrated approach that put together all major physical processes that occur in the neighborhood of the star, the disk, and the $\mathrm{x}$-wind. The SGSL treatment included both internal mechanisms in a wind launched by MHD mechanisms via a star-disk interaction, and external disturbances that could go on top of the background flow. Table 1 in SGSL summarizes the processes that have been built into the approach based on predominately $\mathrm{H}$ atoms: ionizations due to the x-rays, Balmer continuum, $\mathrm{H}^{-}$ detachment, and by collisions; and heating due to X-rays, ambipolar diffusion, Balmer photoionization of $\mathrm{H}, \mathrm{H}^{+}+\mathrm{H}^{-}$neutralization, $\mathrm{H}^{-}$photodetachment, and the mechanical contribution from the environment. The ionization is primarily offset by radiative recombination, and the adiabatic expansion cools very efficiently with the rest: $\mathrm{H}^{-}$radiative attachment, recombination of $\mathrm{H}^{+}, \mathrm{Ly} \alpha$, collisional ionization, and line cooling from the heavy elements.

To mimic the effects of time-variabilities often seen with jets and knots, we adopt here the phenomenological expression for the volumetric rate of mechanical heating (cf. eq [5-2] of SGSL):

$$
\Gamma_{\text {mech }}=\alpha \rho \frac{v^{3}}{s} .
$$

where $\rho$ and $v$ are the local gas density and flow velocity in an inertial frame at rest with respect to the central star, and $s$ is the distance the fluid element has travelled along a streamline in the flow. The coefficient $\alpha \geq 0$ phenomenologically characterizes the magnitude of disturbances, possibly magnetic in origins. A choice of $\alpha \ll 1$ indicates that only a small fraction of the mechanical energy contained in the shock waves and turbulent cascades is dissipated into heat when integrated over the flow volumes of interest at the characteristic distances $s$. Small values of $\alpha$ are required both for self-consistency of a cold flow, and in order to have a regime of weak disturbances on top of a background flow whose properties have been preserved. In the situations where strong disturbances occur, $\alpha$ could and should be very localized in nature, depending on the local dissipation of shocks and turbulence in the neighboring fluid cells. As seen in most jet images, the jets are knotty by appearance, and multi-epoch observations also reveal time-variabilities over the period of various observations. All these possible sources of variabilities and disturbances on the flow add up to an averaged $\alpha$, for modelling purposes. For example, $\alpha \approx 0.002$ would suggest a 
velocity variation less than $5 \%$. Given a typical jet velocity of $300 \mathrm{~km} / \mathrm{s}$, this would imply a weak shock of $15 \mathrm{~km} / \mathrm{s}$ decaying over the jet length. With all the ingredients combined, the thermal profiles thus produced are self-consistent with the underlying dynamical properties, stellar parameters, chemistry, heating and ionizing sources in the framework of star-disk interacting systems.

\section{Jets in Optical Forbidden Lines}

The studies based on SGSL approach suggest that the X-rays and UV photons derived from the accretion processes in young stars could contribute to most of the ionization down deep in the acceleration zone, and that X-rays and recombination on the larger scale, could determine the residual level of ionization in wind and the jet proper over several thousand AU of scale. The level of a few percent and the pattern of reaching a plateau then decaying along the length of jets by recombination is consistent with the trend usually inferred from groundbased and space observations (e.g. Bacciott \& Eislöffel 1999, Bacciotti et al 2000). Figures 1, 2, and 3, in SGSL, illustrate such profiles of ionization on the bottom panels. The top panels in these figures show temperature behaviors for $\alpha=0,0.001$, and 0.002 , respectively. The choice of $\alpha \approx 0.002$ for a slightly revealed fiducial YSO source is the minimum to maintain a temperature high enough in wide enough emitting areas to excite the red forbidden lines. With a temperature around $8,000-10,000 \mathrm{~K}$, the ionization fraction $x_{e}$ holds up very well on the level of few percent on the largest scale. Closer in, $x_{e}$ could rise up to a few tens of percent. Observationally, the highest jet ionization fraction have also been inferred to be from close to the sources (e.g. Lavalley-Fouquet et al 2000, Bacciotti et al 2002). Again, qualitatively, the range obtained from model calculations are within the observational range of 0.02-0.5 (Ray \& Bacciotti 20003).

Synthetic images of fiducial sources can be made by calculating the emission profile with the excitation condition expressed by $x_{e}$ and $T$ (e.g. Fig. 3 of SGSL), and with the cylindrically stratified density structures calculated for with the specific stellar parameters (see Fig. 1 of SSG). The overall brightness in the synthetic image with a temperature profile $T \approx 8000 \mathrm{~K}$ or higher in large enough emitting areas, indeed can be established by a small parameter $\alpha$. Looking at the brightness profile, the projection of a cone-shaped opening near the base actually has appeared, indicating a possible jet opening angle could exist in the optical image out of a wind whose streamlines are launched into all solid angles. The self-consistent excitation profile has changed substantially from the pure column density effect illustrated in Fig. 2 of SSG and suggests that excitation plays crucial roles in how the structure of jets could be revealed. Higher along the length of a jet, the density stratification gradually dominates, and the jetlike appearance stands. This suggests that the excitation conditions reached in jets and winds can completely make over how jets are to be seen optically, and that the effects of heating mainly contribute to change of excitation on top of background flow that could be closely modelled by steady-state values. The optical jets could indeed be a manifestation of "optical illusion" by the interplay of excitation and density collimation. 

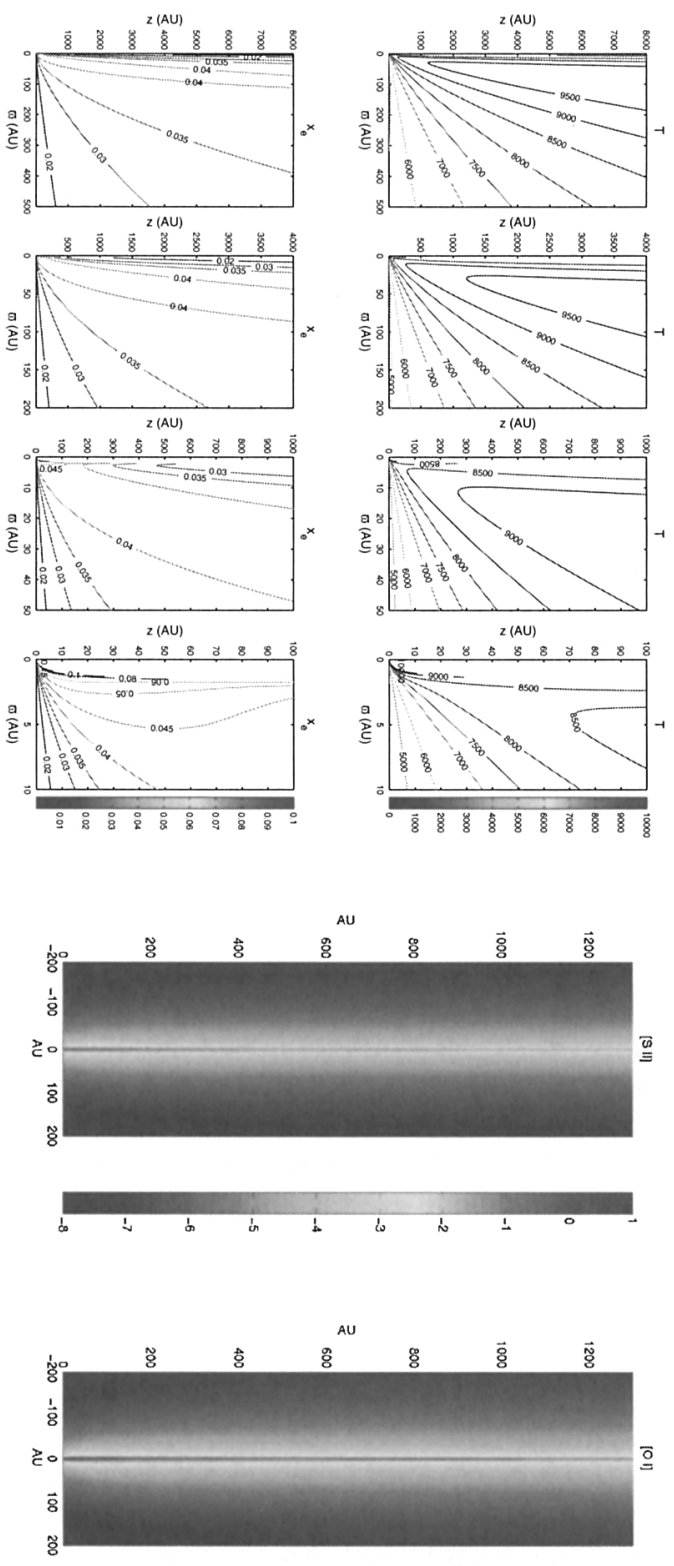

Figure 3. a.(top) Temperature (upper) and ionization (lower) contours in the $\varpi-z$ plane for the fiducial case (defined in Table 2), but with $\alpha=0.002$. The units for the spatial scales are AU. b.(below) Synthetic images of the S II $\lambda 6731$ (left) and OI $\lambda 6300$ (right) brightness for the same model as in a adapting the methods in SSG. The $\log _{10}$ of the integrated intensity is plotted in units of $\mathrm{erg} \mathrm{s}^{-1} \mathrm{~cm}^{-2} \mathrm{ster}^{-1}$. 
The line ratio plots are the best diagnostic tools to ascertain whether such approach could effectively and accurately capture the excitation and physical conditions embedded in jets from optical forbidden line. In Fig. 5 of SGSLwhere the line ratios of S[II]6716/S[II]6731 and S[II]6731/O[I]6300 are plotted against each other. The former is an indicator of the electron density, whereas the latter is an indicator of both the electron density $\left(n_{e}\right)$ and temperature. A cross-correlation plot of these line ratios will uniquely determine the excitation condition by the location on the plot. The small blue dots plotted are each theoretical data point for each "pixel" for the images of S[II] and O[I] jets in Fig. 4 of SGSL. The red stars are data compiled by Raga et al (1996) from ground based observations prior to 1995. Up to the date the plot was made, the pink circles were points for DG Tau with Adaptic Optics (data taken from Fig. 3 of Lavalley-Fouquet, Cabrit, \& Dougados 2002). One can also compare with the AO data for RW Aur (Dougados et al 2002), and the points would fall very well within the coverage. One very noticeable feature of this plot is that the theoretical data dots pretty much encompass all of the observational data points to date. With only few points, whose sources are known to be strong shock excited objects (in knots or bow shocks), lying outside of the loci traced by the shape of potential curves, the physical and excitation condition reached in $\mathrm{x}$-wind jets for a slightly revealed source indeed captures the majority and average conditions inferred from optical jets.

What does such comparison of theoretical and observational data suggest for YSO jets? The treatment of weak shocks works quite well on top of a steadystate background flow. This also suggests that in fact the radiation from jets are mostly excited by weak shocks on average. A single x-wind jet modelled for the slightly revealed YSOs could generate the range of physical and excitation conditions inferred from $\left(n_{e}, x_{e}, T\right)$ of the most studied $\mathrm{HH}$ objects. This also implies that one perhaps can not attempt to label the jets with one single set of numbers $\left(n_{e}, x_{e}, T\right)$ because the system is quite complex. It would require fits from multiple shock curves of various pre-shock densities, velocity variations, and strengths of magnetic fields (e.g. Fig 4. in Dougados et al. 2002). The theoretical models can give a more complete view, and help probe the varieties of conditions.

\section{Jets in Radio Continuum}

Radio jets are elongated, jet-like structures seen at subarcsecond scale. They usually have good alignment with large-scale outflows, usually identifiable with the youngest deeply embedded stellar objects, and have partial association with optical jets or Herbig-Haro objects. In a few YSO sources, the optical jets trace material at scales of several hundred AU and larger, but near the base, small radio jets from ionized material are present very close to the (projected) bases of the optical jets. The typical fluxes from radio jets are weak, around 0.2-2 mJy, and usually are characterized by a small spectral index, $S_{\nu} \propto \nu^{p}$, where $p \geq-0.1$. A complete list of radio jets from both low-mass and high-mass sources, and their respective properties $\left(S_{\nu}, p\right.$, etc) can be found Table 2 in Anglada (1996). 
We are interested in studying low-mass young stellar sources where radio jets have been observed. One of these sources is the archetype L1551-IRS5 found in the Taurus molecular cloud at a distance of $140 \mathrm{pc}$. This source is one of the most studied outflow sources. It has a massive molecular outflow (e.g. Snell, Loren \& Plambeck 1980) driven by an atomic stellar wind (e.g. Giovanardi et al. 2000). L1551-IRS 5 has a bolometric luminosity of $33 L_{\odot}$ and was identified as a Class I source with a total wind mass loss rate of $\sim 2 \times 10^{-6} M_{\odot} y r^{-1}$, obtained from the HI observations (Giovanardi et al 2000). Recently, Rodríguez et al. (2003) used VLA and Pie Town antenna observations with an angular resolution of $0 . " 1$ (14 AU), and found radio jets from the now-identified binary (e.g. Rodríguez et al 1998) at the origin of the larger scale $(\sim 1 ")$ jets observed in both optical and NIR wavelengths (Fridlund \& Liseau 1998; Itoh et al. 2000). The level of radio emission from both jets is $\sim 4 \mathrm{mJy}$, and half of which comes from two compact jet cores.

The SGSL excitation profiles of temperature and ionization, and the electron density distribution for $\dot{M}_{w}=1 \times 10^{-6} M_{\odot} \mathrm{yr}^{-1}, \alpha=0.01$ have resemblance to the profiles shown in Fig. 3 of SGSL. At scales relevant to radio jets, the ionization fraction is on the level of few percent, and this is by a factor of a few reduced from the fiducial case in SGSL at $\dot{M}_{w}=3 \times 10^{-8} M_{\odot} / \mathrm{yr}$. The temperature on the axis near the center is around $10,000 \mathrm{~K}$, as has been inferred for most radio jets. Within $20 \mathrm{AU}$ from the z-axis, electron densities as high as $10^{4}-10^{6} \mathrm{~cm}^{-3}$ can be clearly identified on the scale of 100 AU. Very close in ( $\lesssim 10 \mathrm{AU}$ ), the electron densities could raise even higher to $10^{7}-10^{8} \mathrm{~cm}^{-3}$. As the resolution improves, the higher the electron densities can get closer to the source.

An intensity map at $3.6 \mathrm{~cm}$ based on the above mentioned profile is shown in Figure 4a (and other cases in Shang et al 2003). The contour levels are plotted at powers of 10 . The elongated contours show clearly the collimation due to cylindrically stratified electron density profiles, and can be traced down to $10 \mathrm{AU}$ level. This theoretical intensity map has a lot of fine structure that is yet to be detected by the observation beyond the current 0 ".1 limit. For radio emissions from the jet to be detected by an instrument like VLA at its highest sensitivity and angular resolution, enough total $n_{e}$ and higher mass loss rates with the combinations of parameters are required to produce the right order of magnitudes in total emitted fluxes. The case shown has a total flux from half of jet lobe of $1.14 \mathrm{mJy}$.

A relation of $S_{\nu}-\nu$ (as in Fig. $4 \mathrm{~b}$ ) best illustrates the behavior of total flux with radio frequencies and the spectral index $p$ at various mass loss rates. For mass loss rates lower than $3 \times 10^{-7} M_{\odot} \mathrm{yr}^{-1}, S_{\nu} \propto \nu^{-0.1}$, indicating pretty much transparent emission. The spectra turn over as the mass loss rates progress up to around $\dot{M}_{w} \approx 3 \times 10^{-7} M_{\odot} \mathrm{yr}^{-1}$, at which the indices change from approximately 0.04 , to -0.1 , at around $8.3 \mathrm{GHz}$, suggesting at this mass loss rate emission from optically thick region starts to appear. For mass loss rates higher than the "cross-over" mass rate $\dot{M}_{w}$, the index is approximately 0.3 -which is very close to what has been inferred for L1551.

The extension of the SGSL approach to radio frequencies adds another dimension to the diagnostic tools built upon the SGSL approach and the x-wind model. The radio jets have the following unique advantages. Radio free-free 

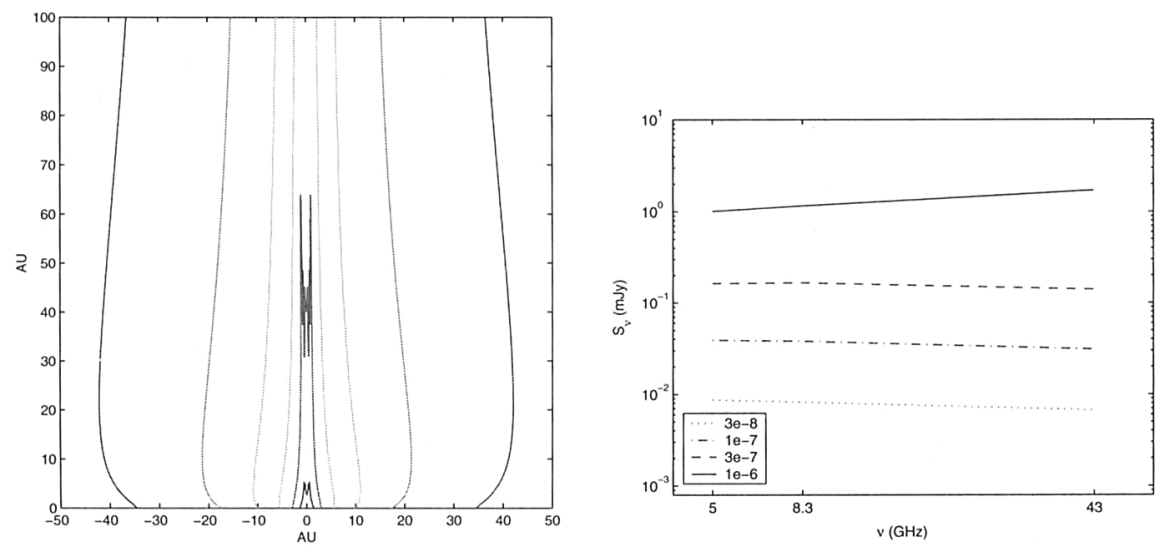

Figure 4. a. Intensity map made for $\dot{M}_{w}=1 \times 10^{-6} M_{\odot} / \mathrm{yr}$, and $\alpha=0.01$. The contours are plotted at powers of 10 , at scales relevant to radio jets. This figure shows more detail than current resolution limit allows one to see. b. A plot of the relationship $S_{\nu}-\nu$ for the four different mass loss rates: $3 \times 10^{-8}, 1 \times 10^{-7}, 3 \times 10^{-7}$, and $1 \times 10^{-6}$ $M_{\odot} / \mathrm{yr}$.

emission at centimeter wavelength is free of dust extinction effects, and no obstruction from disks that the optical and infrared observations and diagnostics usually suffer from. The radio jets have strong connections with deeply embedded protostars and optical jets (if any) from those sources are not easily seen. These make the radio free-free emission a powerful diagnostic tool to probe deeper toward exciting sources.

\section{Processing of Planetary Material}

The magnetic activities resulting from the star-disk interaction at the base of the $\mathrm{x}$-wind has far-reaching implications for the young stars as suns. The complex magnetic structures that strongly resemble the loops, helmet streamers, current sheets on the solar surface do give rise to similar activities analogous to solar activities on an order of magnitude of $10^{3}$ to $10^{4}$ larger in scales and in energies. The loop structure that connect the stellar surface and the edge of the disk have a size typically on the order of $10^{12} \mathrm{~cm}$ in a young stellar system. The twisting and shear due to differential rotation, when the rotational rates between the two footpoints of the field mismatch, will produce elongated and bulged poloidal field and progress possibly in the same fashion as in the loops that are responsible for the gradual flares and the CMEs (e.g. Linker and Mikic 1995). The Y-sheet that lies between the two funnel flows, on the other hand, may simulate the activities and twisting and shearing from smaller loops on the solar surfaces. The current sheet ("reconnection ring" in Figure 1) may have similar activities that are related to the impulsive flares.

The traces of magnetic activities from the outflow phase of our solar system might have been found from the ancient rocks called "chondritic meteorites". On 
the cross-sections of such meteorites such as "Efremovka", one very noticeable feature is the greyish spheroids of a few millimeters to centimeters in size, and rich in calcium and aluminum. These Ca-Al-Inclusions (CAIs) that are usually surrounded by high magnesium content contain the most puzzling mystery to date found in planetary material. Within the CAI phases contain short-lived radionuclides that were once present but now extinct. Among them are ${ }^{7} \mathrm{Be}$, ${ }^{10} \mathrm{Be},{ }^{26} \mathrm{Al},{ }^{41} \mathrm{Ca},{ }^{53} \mathrm{Mn}$, and ${ }^{60} \mathrm{Fe}$, whose origins have been thought to be previous generation stars or internal irradiation by solar system cosmic ray syntheses. The two dramatic lines of interpretation have important consequences in understanding the events that have happened within the first several million years of life for our solar system.

The existence of ${ }^{7} \mathrm{Be}$ (half life of 52 days) and ${ }^{10} \mathrm{Be}$ (half life of $1.5 \mathrm{Myrs}$ ) are the best candidates by irradiation within the early solar system setting. The origins of the cosmic rays may be the magnetic reconnection events in either gradual or impulsive flares associated with the Y-sheets within Figure 1 and 2a. The short half-lives strongly suggest their production within the outflow phase in a situation close to the $\mathrm{x}$-wind setting after the solar system has formed. Within the reconnection ring, where a thin layer of rock material that has drifted in from the accretion disk, cosmic rays can easily interact with rock components and nuclear reactions can take place (Lee et al 1998, Gounelle et al 2001).

On top of the reconnection ring, strong x-rays associated with the magnetic activities and reconnection events can find favorable geometry shinning on the rocks within the reconnection ring that are being irradiated by the cosmic rays. This could suggest one possible mechanism of how the rock material got heated, right within the outflow phase of the evolution (Shu, Shang, Lee 1996; Shu et al 1997).

\section{Summary and Outlook}

We have summarized the most extensive modeling efforts to date in the context of young stars based on $\mathrm{X}$-wind jets with proper heating and ionization. This model can provide overall consistent explanations for general properties of jets in radio free-free emissions for the youngest low-mass stars and in optical forbidden lines from T-Tauri stars, by one systematic approach. This provides a good framework for bringing the theoretical models and observational data together for the outflow phenomena.

The overall $\mathrm{X}$-wind model also provides natural astrophysical setting for the processing of planetary material in the earliest phase of newborn suns. With observational tests on the multiwavelength data, the theoretical model gives predictable physical processes that could further bring the formation and evolution in our solar system into comparison with on-going star formation.

\section{References}

Angalad, G. 1996, in Radio Emission from the Stars and the Sun, eds. A. R. Taylor \& J. M. Paredes, (PASP: San Francisco), 3

Bacciotti, F. \& Eislöffel, J. 1999, A\&A, 342, 717 
Bacciotti, F., Mundt, R., Ray, T. P., Eislffel, J., Solf, J., \& Camezind, M. 2000, ApJ, 537, L49

Bacciotti, F., Ray, T. P., Mundt, R., Eislöffel, J., \& Solf, J. 2002, ApJ, 576, 222

Dougados, C., Cabrit, S., \& Lavalley-Fouquet, C. 2002, RevMexAA (Serie de Conferencias), 13, 43

Dougados, C., Cabrit, S., Lavalley, C., Mnard, F. 2000, A \& A, 357, L61-L64

Fridlund, C. V. M. \& Liseau, R. 1998, ApJ, 499, L75

Giovanardi, C., Rodríguez, L. F., Lizano, S., \& Cantó, J. 2000, ApJ, 538, 728

Gounelle, M., Shu, F., Shang, H., Glassgold, A., Rehm, E., \& Lee, T. 2001

Itoh, Y., et al. 2000, PASJ, 52, 81

Lavalley-Fouquet, C., Cabrit, S., Dougados, C. 2000, ApJ, 356, L14

Lee, T., Shu, F., Shang, H., Glassgold, A., \& Rehm, E. 1998, ApJ, 506, 898

Linker, J. A., \& Mikic, Z. 1995, ApJL, 438, L45

Raga, A., Bhm, K.H., Canto, J., 1996, RevMexAA 32, 161

Ray, T., \& Bacciotti, F. 2003, RevMexAA (Serie de Conferencias), 15, 106

Rodriguez, L. F., et al. 1998, Nature, 395, 355

Rodriguez, L. F., Porras, A., Claussen, M., Curiel, S., Wilner, D., \& Ho, P. 2003, ApJL, 586, L137

Ruden, S. P., Glassgold, A. E., Shu, F. H. 1990, ApJ, 361, 546

Shang, H., Glassgold, A. E., Shu, F. H., \& Lizano, S. 2002, ApJ, 564, 853 (SGSL)

Shang, H., Lizano, S., Glassgold, A., \& Shu, F. H. 2003, ApJ, to be submitted

Shang, H., Shu, F. H., \& Glassgold, A. E. 1998, ApJ, 493, L91 (SSG)

Shu, F., Allen, A., Shang, H., Ostriker, E., \& Li, Z.-Y. 1999, in The Origin of Stars \& Planetary Systems. Edited by Charles J. Lada \& Nikolaos D. Kylafis. Kluwer Academic Publishers, 193

Shu, F., Najita, J., Galli, D., Ostriker, E., \& Lizano, S. 1993, Protostars \& Planets III, 3

Shu, F., Najita, J., Ostriker, E., \& Shang, H. 1995, ApJL, 455, 155

Shu, F., Najita, J., Ostriker, E., Wilkin, F., Ruden, S., \& Lizano, S. 1994, ApJ, 429: 781

Shu, F., Najita, J., Shang, H., \& Li, Z. 2000, Protostars \& Planets IV (Book - Tucson: University of Arizona Press, eds Mannings, V., Boss, A.P., Russell, S. S.), 789

Shu, F., Shang, H., \& Lee, T. 1996, Science, 277, 1475

Shu, F., Shang, H., \& Glassgold, A., \& Lee, T. 1997, Science,

Woitas, J., Ray, T. P., Bacciotti, F., Davis, C. J., \& Eislffel, J. 2002, ApJ, 580, 336 